\title{
IR Thermography Applied to Historical Buildings
}

\author{
by E. Grinzato
}

CNR-ITC, C.so Stati Uniti 4, 35127 Padova, Italy, E-mail: ermanno@itef.pd.cnr.it

\begin{abstract}
Different kinds of discontinuities affecting historical building structures are detectable by thermal analysis of the surface temperature when submitted to suitable boundary conditions. The use of a quantitative approach is illustrated according to particular requirements of works of art. Principal sources of errors and failures in the interpretation of thermographic data are considered.

Applications to massive masonry buildings are reported to illustrate recent results applying advanced processing algorithms to frescoes.
\end{abstract}

\section{Introduction}

IR thermography has been applied for more than 30 years to the buildings monitoring in qualitative or quantitative way $[1,2,3,4,5]$. Even if, both modern and ancient buildings have similar characteristics, cultural heritage needs a lot of care. About historical buildings, the main differences are the limited knowledge of the structure, often modified along centuries, a huge thermal inertia and frequently, the presence of precious parts, as wall paintings. Taking into account the effectiveness and reliability required, the most important demand for a correct thermographic monitoring is the careful design of the inspection procedure. Unfortunately, up to day no dedicated standards, but only a few guidelines are available [6]. A preliminary quantitative study at laboratory level and the use of mathematical simulation of the involved thermal process have been often very useful $[7,8]$. In such a way, thermal analysis makes possible to gather information regarding building and elements technology, their shape, their materials characteristics, and their state of decay.

This paper is mainly devoted in pointing out sources of uncertainties that accompany the experimental procedures. As case study, the inspection of frescos is shown using data processing techniques based on the thermal modelling. Finally, the effectiveness of well known processing algorithms based on the Thermal Contrast can be successfully improved if the structural noise [9] due to the ageing of materials is properly filtered.

\section{Basic Thermography for building inspection}

There are many applications of thermography applied to building science including the microclimate monitoring, non-destructive testing, the moisture mapping, the HVAC system functional, the envelope thermal performance etc. It is a matter of fact that the knowledge of the monument can substantially be improved by the location of hidden structures, as openings or the wall bonding below the plaster [6]. Furthermore, links between the walls is a fundamental information to predict the risk areas for structural weakness. Recently, even mechanical properties of buildings materials have been addressed by Thermography [10]. IR Thermography finds out remarkable results in this field, especially using a quantitative approach. Nevertheless, it is worth noting that the surface temperature itself is a very important parameter for the building investigation. In facts, knowing the temperature distribution is possible for instance to avoid the humidity condensation on walls or evaluating the radiant flux component of the comfort conditions. This not implies that the temperature automatically given by the IR camera is always accurate enough. The main problem is not only the emissivity value or the equipment calibration, but more generally the environment influence, including in this item the camera drift. Many approaches are 
possible and different formulas to get rid of these problems [11]. A reduction of the influence of parameters global changing is achieved adopting a relative approach, even if the selection of a reference is needed. In case of varying surface optical properties, the grey body approximation is considered acceptable for the plaster finishing layer. Hence, a multispectral technique allows a correct temperature measuring; that is acquiring thermograms within two different spectral bands and having a radiometric reference inside the Field Of View (FOV). Such a sophisticate procedure is especially needed when the temperature range is small and the region of interest (ROI) is close to corners [12].

During a thermographic testing, the readout is proportional to the IR flux coming from the scene. The main components of the signal are the thermal radiation emitted by the object (assumed to be an opaque grey body), the thermal radiation emitted by the heater and background reflected from the object surface. This classical configuration leads to relative IR detector output signal $\Delta U(i, j, t)$ of eq. 1 when the thermal radiation from both the atmosphere and IR camera components is negligible.

$$
\Delta U(i, j, \vartheta, t)=c_{1} n \varepsilon(i, j . \vartheta) T_{a}^{n-1}(i, j, t) T_{a a}(i, j, t)+c_{2}[1-\varepsilon(i, j, \vartheta)] \Delta \Phi_{h}(i, j, \vartheta, t)
$$

So, $T(i, j, t)$ is the point temperature, where $(i, j)$ define the pixel coordinates, $t$ is the time, $\theta$ is the viewing angle and $\varepsilon(i, j, \theta)$ is the local emissivity averaged over the spectral range of the IR detector, $T_{a}(i, j, t)$ is the ambient temperature, $\Delta \Phi_{h}(i, j, \theta, t)$ defines the effective energy imposed to any surface element and $c_{1}, c_{2}$, are coefficients related to both the thermographic equipment and the test geometry; $n$ is a coefficient depending on the spectral range of the IR detector ( $n \approx 4 \div 5$ for the $8-13 \mu \mathrm{m}$ and $n \approx 9 \div 10$ for $3-5 \mu \mathrm{m}) . \Delta T_{\text {aa }}(i, j, t)$ is the above ambient temperature difference. It is worth noticing that $T_{\text {aa }}(i, j, t)$ is also a function of $\Delta \Phi_{h}(i, j, \theta, t)$ thus, involving both thermal and optical parameters. Generally, a certain amount of radiation is reflected far from the surface and contributes to the so call apparent temperature. A useful tool to get rid of this problem is a passive reference, placed in the field of view, indicating both the room temperature (looking at a central cavity) and the environment component (looking at a reflective-diffusive frame)[13].

Because generally we are interested to indirect measures, the determination of the searched unknown physical parameters involves the following scheme:

IR imager signal $\Rightarrow$ Sample true temperature $\Rightarrow$ Mathematical model $\Rightarrow$ Unknown parameter.

The effectiveness of the test depends mostly on the following items:

- the proper equipment adopted, including not only the thermal camera but also the needed heat sources and appropriate supporting frame;

- the most suitable testing procedure, along with the optimal environmental conditions, the taking site, the timing requirements, the needed ancillary measurements and the recording of data.

- the right processing algorithm (in the following Thermal Tomography is used);

- the output, usually produced assembling and cross-analysing different elementary results.

The use of a quantitative approach allows great advantages, as the higher reliability of findings, but it is much more time consuming. In facts, most of algorithms involve the time analysis, processing many thermograms for any examined FOV instead of a single IR image. Actually, active techniques are more expensive because they need a set of suitable heat sources to be placed close to surfaces, often critically settled. Therefore, the chosen approach must be carefully evaluated accordingly to the particular target and requirements.

\section{Experimental procedure and mathematical modelling}

Mathematical modelling of the heat and mass transfer is not trivial, due to the complexity of the structure and materials uncertainty. Hence, the problem must be 
strongly simplified in order to achieve practical results and avoid the output of the simulation become richer of question marks than statements. A good cost/benefit compromise is the analysis of selected parts of the structure using specialised packages [14]. Notice that an analytical model could help only in limited situations, when the geometrical structure is very simple and thermal parameters do not vary with time or temperature. Nevertheless, a comparison of analytical and numerical simulation allows to verify accuracy of results.

Usefulness of mathematical simulation is related to the agreement between the experimental and computed temperature evolutions. Discrepancy depends on many factors, but formerly the thermal modelling needs true thermal properties values and the actual boundary conditions. Furthermore, a precise description of the building geometry is complex and sometime impossible. In practice, it is hard to fulfil previous issues, especially for historical buildings due to large variability of situations. The analysis of principal sources of errors was performed simulating a fresco section submitted to typical conditions during a non destructive thermographic test (TNDT). The goal of the test is the evaluation of the plaster adhesion, as schematised in the 3D geometrical model of Fig. 1. The surface temperature pattern vs. time has been computed mainly by off the shelf finite difference or finite elements (FEM) codes [15]. By comparison with an analytical model, errors in numerical modelling have been estimated less than $0.5 \%$ in sound areas and about $1-5 \%$ over finite-size subsurface defects. The fresco was considered as constituted of two layers: the plaster $\left(k=0.48\left[\mathrm{~W} \mathrm{~m}^{-1} \mathrm{~K}^{-1}\right]\right.$, thermal diffusivity $\left.\alpha=0.2310^{-6}\left[\mathrm{~m}^{2} \mathrm{~s}^{-1}\right]\right)$ and a supporting wall made by bricks $\left(k=0.7\left[\mathrm{~W} \mathrm{~m}^{-1} \mathrm{~K}^{-1}\right]\right.$, thermal diffusivity $\alpha=0.5210^{-6}\left[\mathrm{~m}^{2} \mathrm{~s}^{-1}\right]$ ) or stone $\left(k=2\left[\mathrm{~W} \mathrm{~m}^{-1} \mathrm{~K}^{-1}\right]\right.$, thermal diffusivity $\left.\alpha=0.8810^{-6}\left[\mathrm{~m}^{2} \mathrm{~s}^{-1}\right]\right)$. Experimental data are compared in fig. 2 with the computed one for different options. Thermal conductivity was decreased by $10 \%$ and doubled the heat exchange coefficient ( $h=5$ or $h=10\left[\mathrm{~W} \mathrm{~m} \mathrm{~K}^{-1}\right]$ ), without appreciable effects [15]. Even the consequence of constant or variable $h$ values has been evaluated in such a way, including both convection and radiation. In the case of heat exchange intensity varying with temperature a very similar result came up. The comparison between the adiabatic and non-adiabatic models is illustrated in fig. 3 by the analytical modelling of an infinite slab. Useful guidelines can be given by using dimensionless parameters as the Fourier number $\left(F_{o}=\alpha t \mathrm{~L}^{-2}\right)$ instead of time $t$. Computations illustrated by fig. 3 have been performed for different length of the heating (150 and $300 \mathrm{~s})$ and Biot numbers $\left(B i=h L K^{-1}\right)$ is typically of the order of 0.1 . Hence, the adiabatic solution cannot be used for frescos.

Often, the wall construction is unknown and local plaster thickness $(L)$ can reach 20 $\mathrm{mm}$ or more. It is well known that in conventional TNDT the plaster affects more the surface temperature than the masonry. Sensitivity to this parameter can be estimated by analysing the behaviour of the $(\Delta T / T)(\Delta L / L)^{-1}$ function. The curves of Fig. 4 illustrates influence of plaster thickness, having been obtained for two heating times $\left(t_{h}=150 \mathrm{~s}\right.$ or $300 \mathrm{~s}$ ), that is $F_{O_{\mathrm{h}}}=0.05$ or $F_{O_{\mathrm{h}}}=0.1$. The surface temperature starts to 'sense' inner interfaces (i.e. the plaster thickness $L$ ) after a certain time from the thermal excitation. For a $20 \mathrm{~mm}$ plaster layer, the time during which surface temperature is not significantly influenced by the thickness corresponds to $F_{\mathrm{O}_{\mathrm{h}}}=0.2$, that is $t<400 \mathrm{~s}$. Notice that this time can be shorter than the heat pulse. Later on, the sensitivity to the layer thickness is quite significant. Negative sign of $(\Delta T / T)(\Delta L / L)^{-1}$ indicates that the surface temperature decreases as thickness increases. In other words, for times shorter than $400 \mathrm{~s}$ the model of a semi-infinite body fits well, whether the heating device is switched on or not.

Eq.(1) indicates as both a thermal component (through the $T_{a a}(i, j, t)$ term) and an optical one (given by the imposed heat flux $\Delta \Phi_{h}(i, j, \theta, t)$ contributes to the output signal of the IR system. Thermal and optical phenomena can be hardly separated because heating is often performed with optical sources and temperature is measured by IR radiation. The influence of optical parameters is concentrate normally in the role of emissivity, but often underestimated for others aspects. This statement is true indeed for active TNDT, where 
defects looks as uneven heating pattern. It is worth mentioning also that the hot heaters gives "post-heating tails" even after they have been switched off. The fig. 5 shows the surface temperature increasing during a TNDT if the heat source has been covered by a shutter or not. The higher radiation collected by the infrared camera during the cooling phase is not taken into account by the mathematical modelling. At the meantime, changing the shape of the heating function vs. time modifies the surface temperature evolution. For example, in the case of square pulse heating, the maximum surface temperature occurs at the end of heating. But injecting the same amount of energy $(Q)$ with a triangular or cosine-shaped pulse, as long as the square pulse, the highest temperature will be within the heating time. Fortunately, it has been demonstrated [16] that in practice the heating function vs. time has importance only for early times of the cooling phase and the amount of energy is proportional to the signal to noise ratio. Thus, if the optimum observation time occurs during the cooling phase, only the total absorbed energy affects linearly the temperature rise. Optical parameters of interest are therefore both the spectral irradiation and the local absorption coefficient. A very useful technique to bypass the knowledge of such parameters is a relative processing. The thermal signal $\Delta T$ is the surface temperature variation vs. a reference assumed to be representative of the sound material. A truthful reading of the thermal signal needs a compensation for certain factors as the surface optical features. For this purpose, the maximum temperature is an important normalisation factor. Unfortunately, normalisation as any ratio, even if reduces significantly low frequency signals caused by the uneven heating, tends to introduce a high-frequency noise. Furthermore, the usefulness of normalisation decreases for any subsequent image in a sequence because of the changing in the boundary conditions and non-linearity of radiative processes. So, the unevenness is not completely corrected by maximum temperature normalisation, also because of the 3D heat diffusion generated by temperature gradient. It is worth mentioning the colours on the surface give inhomogeneous energy absorption and work differently in the different spectral bands. Using a 3D heat transfer model allows to take into account the heating variation in time and space. Applying a Q-mask, as shown in fig.1, it could be simulated the full effect of the uneven heating. The Q-mask is the distribution of the heat flux density imposed at each surface point. Such a $Q$-mask is derived from the experimental data using a thermogram taken at a very early time. The following paragraph 4 illustrates in more detail this issue.

There are in the practice ambiguous cases where the use of a multilayer modelling of the thermal problem helps a lot the analysis. For examples, overlapping defects placed at different depth, or the presence of spurious material used for previous restoration activities are common. Figure 6 reveals the scheme of two overlapping air gaps embedded in a plaster layer and the surface temperature due to their superposition. The synthetic thermograms computed at 80 and $260 \mathrm{~s}$ after the end of an imposed heat flux show the footprint of both defects. The temperature profiles along the thermogram allow to quantify the thermal signal. It can be stated, as a rule of thumb that for most building materials overlapping defects follows a superposition law with an error less than $10 \%$.

A second example exploiting mathematical modelling is illustrated by fig.7 where a thermogram taken over a fresco submitted to a TNDT shows both warmer and cooler spots. Here, inclusions are supposed to be made of air (left column) or resin (right) and such hypothesis has been established simulating the temperature field by FEM. The reported temperature cross sections have been computed using boundary conditions and geometry similar to the test. They demonstrate as the cool areas are due to the more capacitive resin injections used for a restoration, while the warmer areas are due to resistive air inclusions. At the meantime, the depth of inclusions has been evaluated by comparison between experimental and computed plots vs. time of thermal contrast. In facts, the second row of fig. 7 shows different contrasts changing the depth of inclusions. 


\section{Enhanced Thermographic data reduction procedures}

The international literature frequently presents optical techniques suitable for testing of valuable paintings. This target seems to fit perfectly potentialities of IR thermography. Unfortunately, the presence of the painting on the surface makes the analysis much more difficult as anticipated in the paragraph 3 . In particular, lateral heat conduction generates false alarms and artifacts when data are processed using a simplified 1D model. This still open problem, is approached merging experimental data with synthetic thermograms given by mathematical simulation of TNDT [17]. In 1998, along with an intensive restoration of the Malpaga castle (BG Italy), we inspected a large number of frescoes, monitoring the adhesion status. The TNDT was fulfilled warming several $0.92 \times 0.46 \mathrm{~m}$ sections of the fresco. A special frame supported four quartz lamps (1500 W each) and the IR camera operating in the 8-13 $\mu \mathrm{m}$ band (see Fig. 8a). Image sequences were recorded at a rate of $10 \mathrm{~s}$ for $900 \mathrm{~s}$ starting at room temperature, including the heating phase lasting for $150 \mathrm{~s}$ and the cooling phase as well. Results given by Thermal Tomography has been considered good by the restoration authority, but in many cases only the knowledge of the monument and alternative NDT methods clarify some doubts [18]. The most important steps of data treatment involved: 1) subtracting the initial image at room temperature from the rest of the sequence in order to consider only above room temperature, 2) normalising the sequence by the image taken at the end of heating (150 s), 3) selecting a sound reference area where the tap test revealed no subsurface defects, 4) compute for any pixel the thermal contrast, 5) producing a couple of images called 'maxigram' (maximum contrast) and 'timegram' (time of maximum contrast), 6) synthesising thermal tomograms and producing the 'depthgram' and the 'thicknessgram'. using individual calibration functions [19]. The repeatability of temperature measurements from one test to another was not worse than $0.2^{\circ} \mathrm{C}$, within a $95 \%$ confidence level when the fresco was cooled down to the ambient temperature before the next test. The accuracy of determining the normalised temperature contrast is about $5 \%$. This is reasonable because of the minimal temperature increase allowed for inspecting frescos.

The same bunch of data has been used later on to improve the analysis using more sophisticated algorithms [20]. Fig. 8b shows a fresco section chosen to illustrate enhancement given by taking into account the 3D heat diffusion. The idea is the extraction of defects signature comparing each experimental thermogram with a synthetic sequence computed according to the scheme of fig.1, but without any defect. The computation must reproduce temperature field and the evolution in time in 3D within a combined thermal/optical model. The accuracy of the numerical model on the surface of the fresco ranges from 1 to $6 \%$. For fresco and plaster $k=0.23\left[\mathrm{~W} \mathrm{~m}^{-1} \mathrm{~K}^{-1}\right], \alpha=0.2110^{-6}\left[\mathrm{~m}^{2} \mathrm{~s}^{-1}\right]$ and for air $k=0.07\left[\mathrm{~W} \mathrm{~m}^{-1} \mathrm{~K}^{-1}\right], \alpha=5810^{-6}\left[\mathrm{~m}^{2} \mathrm{~s}^{-1}\right]$. The heat exchange coefficient was $h=6\left[\mathrm{~W} \mathrm{~m}^{-2} \mathrm{~K}^{-1}\right.$ ]. The maximum above ambient temperature difference in a sound area predicted by the model was $7.4^{\circ} \mathrm{C}$. This was close to the experimental value, which was $7.6^{\circ} \mathrm{C}$. On the top of the image $8 \mathrm{~b}$ can be seen the feet of the characters and a horizontal grey band give areas of different energy absorption. At the mean time, corners are systematically warmer on the whole sequence due to uneven irradiation. The normalisation process for the maximum temperature reduces effects of uneven heating but do not cancel the heat flux parallel to the surface. The two thermograms shown on the left column of fig. 9 has been chosen to illustrate the technique. The upper row of fig.9 deals with the end of heating $(150 \mathrm{~s})$ and the thermogram of second row has been taken at the optimum time for the detachment detection (600 s). Thermal profiles corresponding to the column marked on thermograms are shown in the right column of fig. 9 together with the computed one. Experimental and computed profiles are in good agreement and simulated data are less noisy, because of a proper meshing. More important, only when a defect exists we observe a deviation of temperatures. Figure 10, shows a comparison of the raw thermogram at $600 \mathrm{~s}$ (left), the computed one (middle) for the same conditions and the result of the processing (right). The purpose of removing artefacts due to the uneven heating is here fulfilled and processing this sequence with the same algorithm of Thermal 
Tomography will give a much better result. It is worth noting that computed sequences act as a reference, not requiring any operator intervention for the processing.

Finally, a noise filtering procedure recently introduced, but well known since a long time is the fitting of data in time domain [21]. This approach is particularly useful for historical buildings for two reasons: the level of the noise due to the structure itself is very high, the thickness of structures makes reasonable the approximation of a semi-infinite body. Therefore, the linear representation of the $\operatorname{Ln}(T)$ in the $L n(t)$ scale $(L n-L n)$ is suitable. Generally, a polynomial fitting in the $L n-L n$ space is more appropriate when defects or hidden structures exists. [22]. Figure 11 gives an example of this procedure showing temperature plots fitted using a $4^{\text {th }}$ order function. Differences between the raw and fitted temperature are of the order of $10^{-4} \mathrm{~K}$. The identification of maximum of thermal contrast after the polynomial interpolation is much easier, even for deep defects. Another feature of this procedure is the dramatic data reduction because the whole sequence is condensed in a few images giving the coefficients maps $A_{k}$, indicated in the eq.2.

$$
T(i, j, t)=\prod_{k=0}^{n} e^{A_{k}[\ln (t)]^{n}}
$$

Sometime, the maps of coefficients $A_{k}$ gives themselves a qualitative indication of defects, but the physical interpretation is not totally clear. For sure it is highly desirable such a noise filtering before any further processing and particularly Thermal Tomography where the maximum contrast is very affected by the noise. In facts, figure 12 shows the original timegram (left) and the timegram after the $L n-L n$ processing (right) for a fresco region where resin has been injected during previous restoration. Here, the spot with indications of detachment and resin insert are respectively classified by red or cyan colour.

\section{Conclusions}

Thermographic monitoring of historical buildings is challenging from both practical and academic points of view. Among different applications, the correct temperature mapping is the starting point. Many aspects have to be taken into account due to the complexity of the structure and the interaction of different energy fluxes. The use of a fully quantitative approach seldom matches time and budget requirements, but it is extremely important in order to solve ambiguous cases. Even if a passive qualitative monitoring is chosen, mathematical modelling of the thermal problem allows to decide if and when thermography is appropriate to a particular case. As a matter of fact, the strong heat diffusion allows to detect subsurface structures or voids, accessing only one surface, up to a depth of a few centimetres.

Mathematical modelling is very useful also for setting up the testing procedure, developing new algorithms and improving diagnosis. Therefore, quantitative thermography is expanding its applications to historical buildings and works of art. The combination of thermal and optical phenomena strongly influences the inspection results. Hence, temperature history should be normalised in order to reduce the influence of absorbed energy. An accurate heat conduction model is relatively simple except the knowledge of materials and geometry is required. The discrepancy between experimental and calculated data mainly comes from the uncertainty in input parameters, such as absorbed energy, heat exchange coefficient, heating function, shape and thermal properties of materials. Analysing individual source of errors indicates how it can be minimised by proper modelling. Heat pulse shape is not an important factor if signal observation is made within the fully developed cooling stage. Furthermore, the thermal diffusivity of building materials does not influence the shape of normalised temperature curves, and the plaster rear-surface morphology is not sensed if the observation time is properly chosen. The optical effect resulting from the heater radiation reflected back from the fresco surface can be significant, but diminished by shuttering the heater. The main factor limiting the 
potential of TNDT is believed to be the uneven energy absorption and the following 3D heat diffusion. In fact, the presence of the surface clutter, including the fresco itself, leads to plentiful false defect indications in both original and processed images. For instance, in dynamic thermal tomography, these false indications appear as crown-like footprints corresponding to zones placed at edges of different absorption areas.

As case study, it is shown the improvement of NDE obtained for the fresco inspection. It is possible to reduce $3 D$ heat diffusion phenomena comparing image-by-image the experimental sequence with its computed replica, but defect free. This technique has provided a nearly three-fold increase in the signal-to-noise ratio. Enhanced results have been achieved also by means of polynomial fitting in the $L n-L n$ scale. Thermograms have been processed before applying thermal tomography for the defects characterisation. $L n$ $L n$ data fitting allows an effective noise filtering and a significative data compression.

\section{Acknowledgements}

This paper it should not be possible without the long lasting cooperation with prof. $V$. Vavilov, dr. S. Marinetti and dr. P. Bison.

\section{REFERENCES}

[1] LJUNGBERG S.A., "Techniques in Buildings and Structures. Operation and Maintenance", Infrared Methodology and Technology, Non-destructive Testing Monographs and Tracts, Vol.7, G. Maldague Ed., Gordon \& Breach Science Publishers, U.S.A., 1994, p. 211-252.

[2] CHIELDS K.W., COURVILLE G.E. and CHIELDS P.W., "An investigation of factors influencing Infrared roof moisture surveys using a mathematical model." Thermosense VI, Orlando (USA), SPIE vol. 446 1983, p.82-94.

[3] BÜSCHER K.A., WIGGENHAUSER H. and WILD W., "Infrared Optical Moisture Measurements in Building Materials Using Amplitude Sensitive Modulation Thermography" $5^{\text {th }}$ AITA, Venice 1999, p. 157-165.

[4] GRINZATO E., BISON P.G., MARINETTI S. and VAVILOV V., "Non-destructive evaluation of delaminations in fresco plaster using Transient Infrared Thermography", Research in Nondestructive Evaluation, Springer-Verlag, New York, vol.5, 4, 1994, p.257-271.

[5] E. GRINZATO, P. G. BISON and MARINETTI S., "Monitoring of ancient buildings by the thermal method", Journal of Cultural Heritage", Elsevier, 3, 2002, p. 21-29

[6] ASNT Nondestructive Testing Handbook, third edition: Volume 3, Infrared and Thermal Testing, Technical Editor: Xavier P.V. Maldague. Editor: Patrick O. Moore, 2001, cap. 18

[7] VAVILOV V., KAUPPINEN T. and GRINZATO E., "Thermal charactersation of defects in building envelopes using long square pulse, and slow thermal wave techniques"; Research in Nondestructive Evaluation, Springer-Verlag, New York, vol. 9, 1997, p. 181-200

[8] E. GRINZATO, BRESSAN C., PERON, F. P. ROMAGNONI and A.G. STEVAN: "Indoor climatic conditions of ancient buildings by numerical simulation and thermographic measurements", Thermosense XXII ${ }^{\circ}$, 2000, SPIE vol.4020, p. 314323

[9] GRINZATO E., VAVILOV V., BISON P.G., MARINETTI S. and BRESSAN C.: "Methodology of processing experimental data in Transient Thermal NDT", Thermosense XVII', SPIE vol. 2473, 1995, p. 167-178

[10] LUONG M.P. "Infrared detection of thermomechanical coupling in solids", SPIE Vol 4710, 2002, p.492-506

[11] MALDAGUE, X.P.V., "Nondestructive evaluation of materials by infrared thermography", Springer-Verlag, London, 1993, 184, p. 1993 
[12] GRINZATO E., BRESSAN C., MARINETTI S., BISON P.G. and BONACINA C. "Monitoring of the Scrovegni Chapel by IR Thermography: Giotto at Infrared"; Journal of Infrared physic and technology, vol.43, 2002, p.165-169

[13] OHMAN C., "Practical methods for improving thermal measurements", Thermosense IV ${ }^{\circ}$ SPIE vol.313, 1981, p.204-212

[14] ROSINA E., GRINZATO E. and ROBISON E,,: "Mapping hidden wall structures by Quantitative IR Thermography"; Thermosense XXIV ${ }^{\circ}$, SPIE vol.4710, 2002, p.253264

[15] VAVILOV V., KOURTENKOV D., GRINZATO E., BISON P.G., MARINETTI S. and BRESSAN C., "Inversion of experimental data and thermal tomography by using "Termo.Heat" and "Termidge" software",. Eurotherm Seminar \#42, QIRT 94, 1994, p.273-278

[16] VAVILOV V., MARINETTI S., GRINZATO E., BISON P.G., DAL TOE S. and BURLEIGH D., "Infrared Thermographic Nondestructive Testing of Frescos: Thermal Modelling and Image Processing of Three Dimensional Heat Diffusion Phenomena", Material Evaluation, vol.60, 3, 2002, p.452-460

[17] GRINZATO E., BISON P.G., BRESSAN C. and MAZZOLDI A., "NDE of frescoes by Infrared Thermography and lateral heating"; Eurotherm Seminar n. 60, QIRT 98, 1998, p.64-67

[18] GRINZATO E., BRESSAN C. and MAZZOLDI A., "The quantitative IR Thermography for the diagnosis of frescoes", $4^{\text {th }}$ International Workshop on Advanced Infrared Technology and Applications, 1997, p.345-366

[19] VAVILOV V., GRINZATO E., BISON P.G., MARINETTI S. and BRESSAN C. "Thermal Characterisation and Tomography of Carbon Fibre Reinforced Plastics Using Individual Identification Technique", Material Evaluation, vol.54, 5, 1996, p.604-610

[20] GRINZATO E., BISON P.G., MARINETTI S. and VAVILOV V., "Thermal NDE enhanced by $3 D$ numerical modeling applied to works of art", Insight vol. 43, 4, 2001, p.254-259

[21] BALAGEAS D.L., KRAPEZ J.-C., and CIELO P., "Pulsed photothermal modeling of layered materials", J. Appl. Phys., Vol.59, 2, 1986, p.348-357

[22] SHEPPARD S.M., LOTHA J.R., RUBADEUX B.A., AHMED T. and WANG D., "Enhancement and reconstruction of thermographic NDT data", Thermosense XXIV ${ }^{\circ}$ SPIE vol.4710, 2002, p.531-535

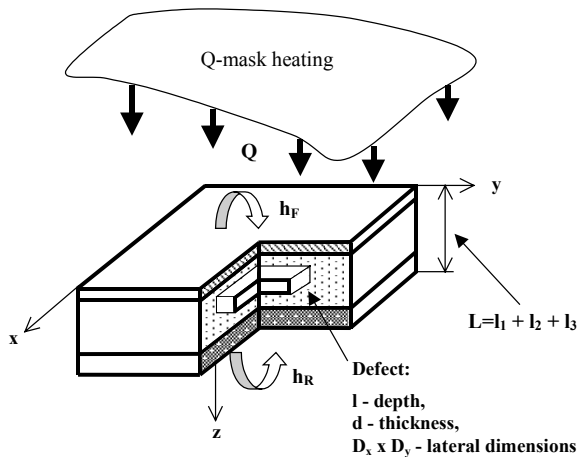

Figure 1. Model of the multidimensional thermal simulation for the inspection of fresco (11, I2, 13 are different buildings materials)

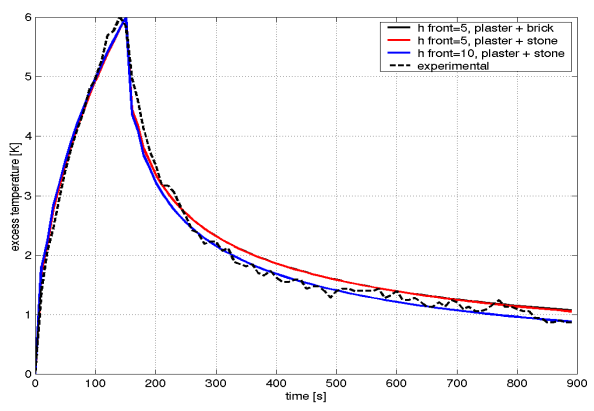

Figure 2. Surface temperature compared with simulation results inspecting a sound fresco for different material and heat exchange options 


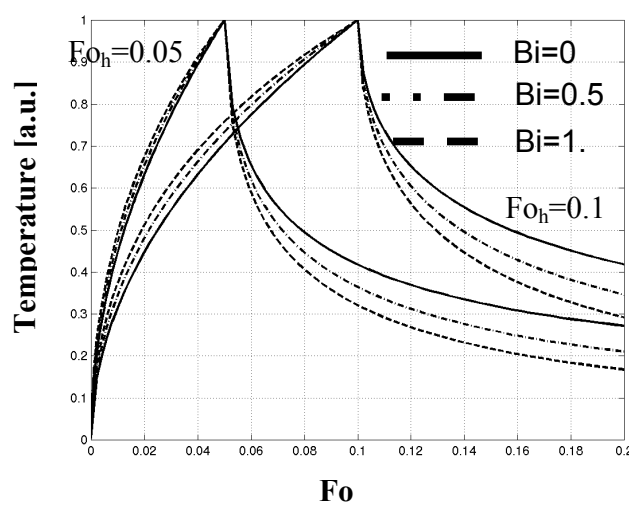

Fig. 3. Analytical simulation for two heating duration in adiabatic and non-adiabatic conditions (plaster inspection)

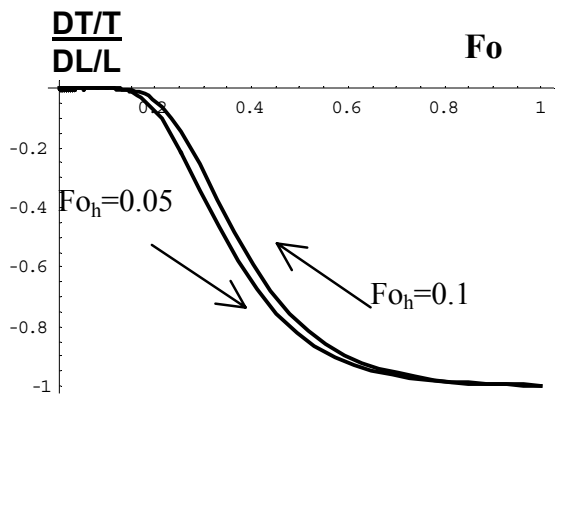

Fig. 4. Influence of sample thickness on the front surface temperature evolution (adiabatic plate, square pulse heating)

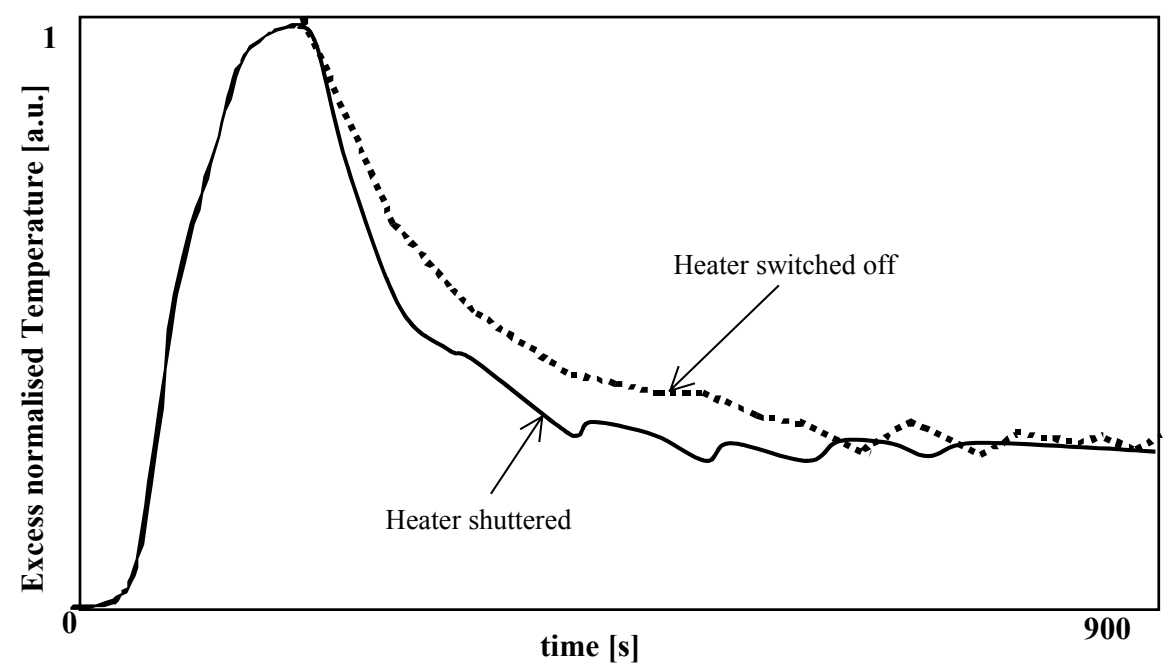

Fig.5. Surface temperature history with and without the strain radiation from the heater 

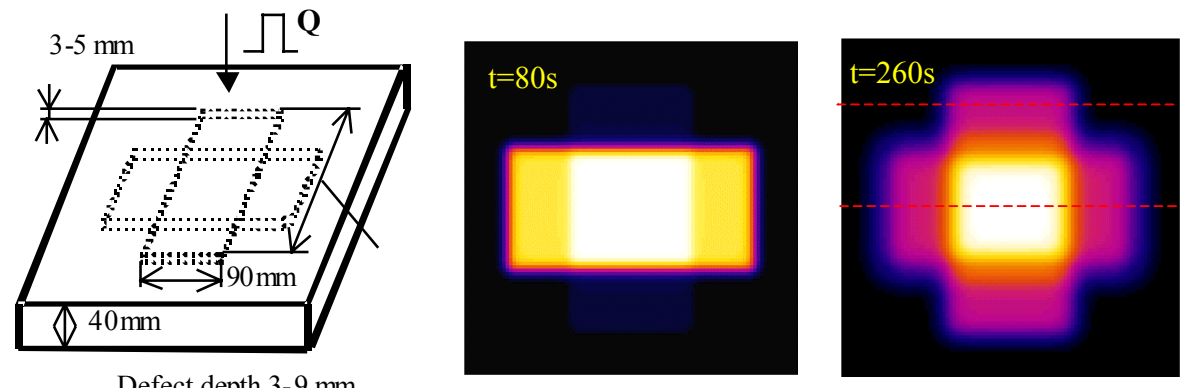

Defect depth 3-9 mm
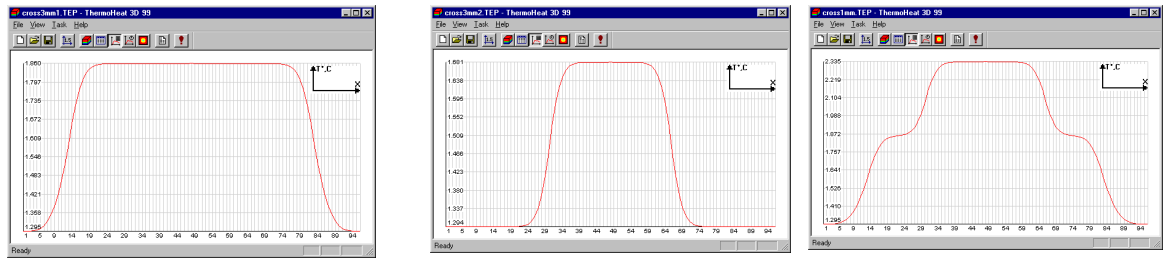

Fig. 6. Draw and simulation of overlapping defects verifying the superposition principle; computed thermograms at 80 and $260 \mathrm{~s}$, the second row shows thermal profiles for shallow (left), deep (middle) and overlapping defects (right) at $260 \mathrm{~s}$
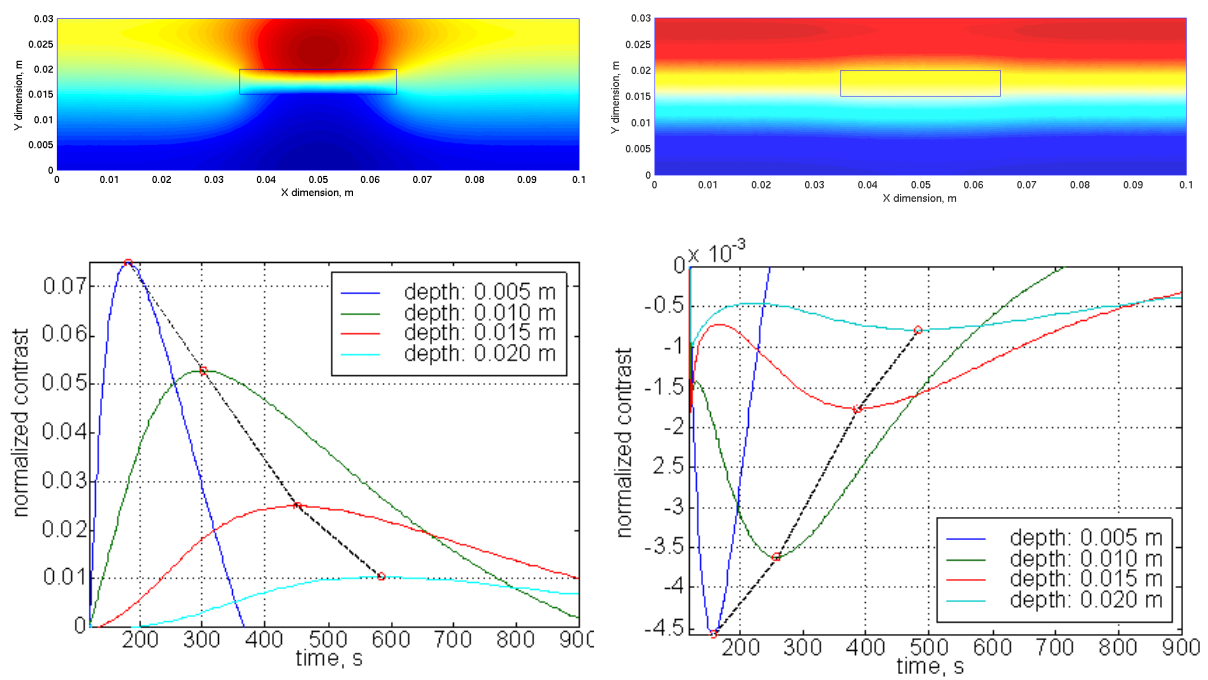

Fig. 7. FEM Simulation of temperature due to air (left) or resin (right) inclusions in $30 \mathrm{~mm}$ plaster;

first row: cross section thermogram for defects at $10 \mathrm{~mm}$ depth at the time of maximum contrast,

second row: normalised contrast vs. time of surface temperature for different defects depth 


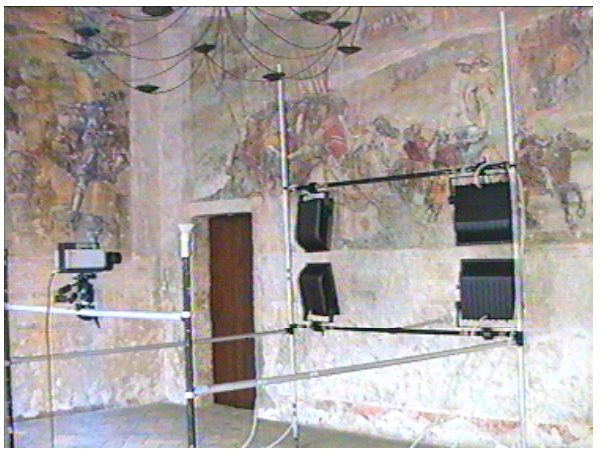

Figure 8a. The experimental set-up for TNDT

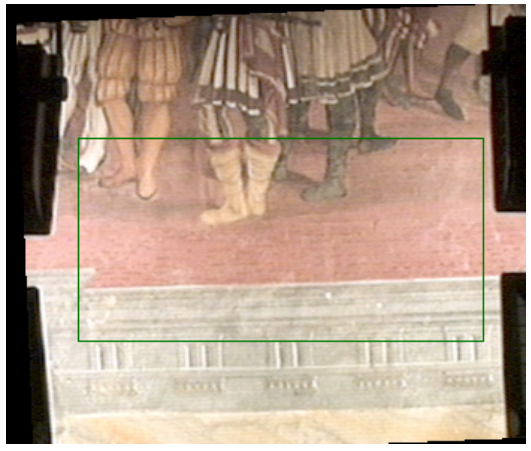

Figure $\mathbf{8 b}$. A tested area (inside the box)
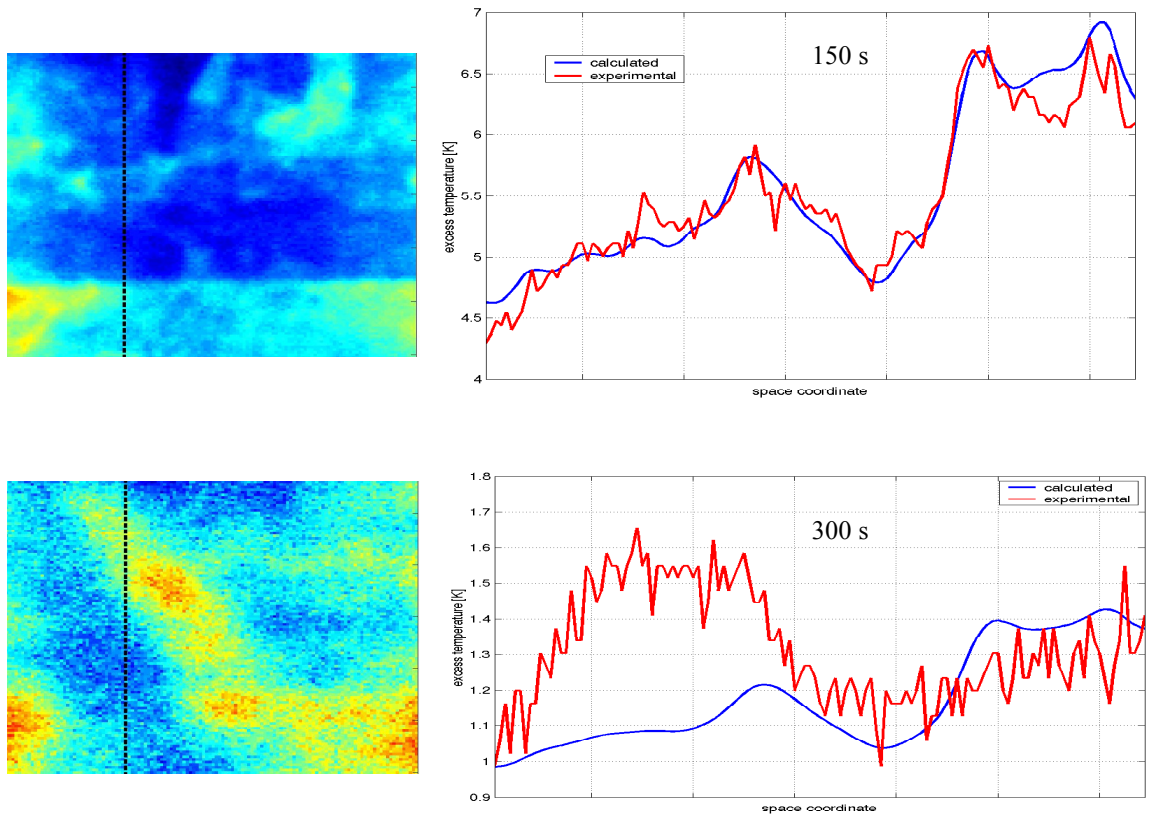

Fig. 9. Comparison of experimental and simulated thermal profiles along the column marked on the thermograms at 150 and 300 s in the inspection of the a fresco 

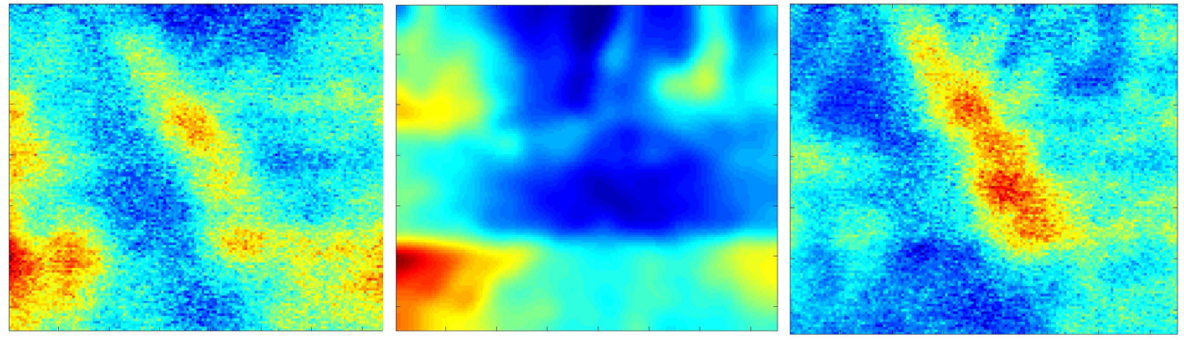

Fig. 10. Filtering of thermograms for the $3 D$ heat diffusion: raw, simulated and processed one
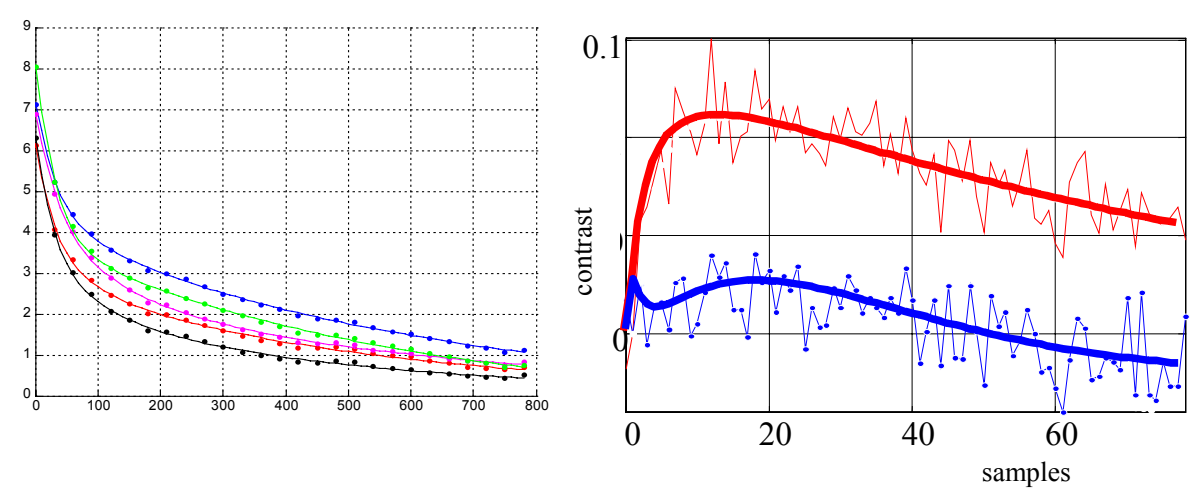

Fig.11. Noise filtering by linear fitting in the In-In scale of raw temperature data for fresco NDT and normalised contrast profiles after reconstruction using a $4^{\text {th }}$ order polynomial function
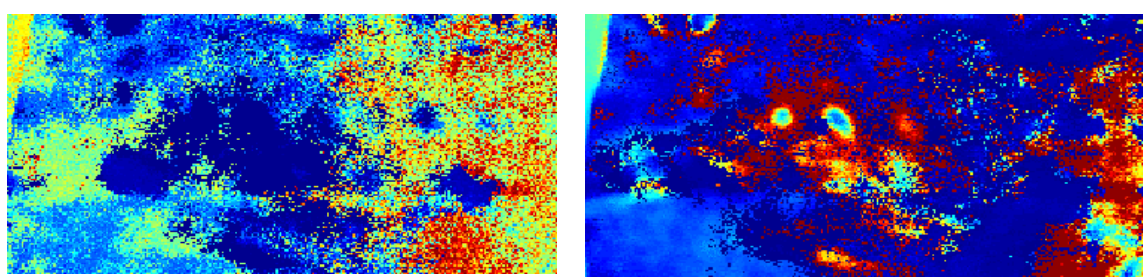

Fig.12. Thermal Tomography enhanced by the noise filtering using In-In fitting: timegram executed on raw data (left) and after reconstruction using a $4^{\text {th }}$ order polynomial function (right) 